\title{
A Novel 3D Reconstruction Algorithm of CT Images Based on Improved Marching Cubes Algorithm
}

\author{
Dongxue $\mathrm{Su}$, Lin $\mathrm{Ma}^{*}$ \\ hitsudongxue@163.com,malin@hit.edu.cn \\ School of Electronics and Information Engineering, Harbin Institute of Technology, Harbin, China
}

\begin{abstract}
In 3D reconstruction for CT images, the Marching Cubes (MC) algorithm is a popular used surface rendering algorithm. However, current MC algorithm has to calculate a large amount of data and triangular patches, which leads to a lower speed of the algorithm. This paper proposes an improved MC algorithm by improving the representation of voxels. By increasing the volume of voxels, the number of voxels that need to be traversed in $3 \mathrm{D}$ reconstruction is greatly reduced. Before the isosurface is drawn, the triangle mesh is subtracted. At the same time, in the process of calculating the equivalent points, this paper proposes to introduce smoothness and distortion coefficients to control the smoothness and distortion of the 3D reconstruction model. Simulation results show that the proposed algorithm is more efficient than the MC algorithm, and improves the real-time interaction and the controllability of smoothness and distortion.
\end{abstract}

Keywords: Marching Cubes algorithm;CT image;3D reconstruction

\section{Introduction}

In recent years, with the development of modern medical imaging technology, computer tomography (CT), magnetic resonance imaging (MRI), ultrasound (US) and other medical imaging technologies have been widely used. In the current medical diagnosis, the doctor creates a 3D model in his mind by observing the CT sequence pictures. And make corresponding treatment plans with their own experience. However, this approach obviously increases the difficulty for doctors to diagnose. The accuracy of the diagnosis made in this way depends largely on the clinical experience of the doctor. It is difficult to reach an accurate judgment. In order to reduce the negative impact of subjective judgment and insufficient clinical experience on diagnosis, medical image segmentation and 3D reconstruction are performed with the help of computers.

Generally, 3D reconstruction methods are mainly divided into two categories: 3D surface rendering and 3D volume rendering. Volume rendering operations are huge and cannot meet the needs of clinical interaction. Therefore, surface rendering is still the mainstream of clinical $3 \mathrm{D}$ reconstruction. Surface rendering is the reconstruction of intermediate geometric units from the original 3D medical image data. The geometric unit is used to describe the 3D structure of the object. In 3D reconstruction of medical images, the main algorithm used for surface rendering is the Marching Cubes (MC) algorithm proposed by Lorensen [1,2]. Its essence is to extract an isosurface from a 3D data field. So it is also called isosurface extraction algorithm. At present, with the development of medical equipment, medical image 
data sets are getting larger and larger. Massive data brings huge challenges to traditional 3D reconstruction techniques.

The MC algorithm needs to extract a large number of triangular patches included in the surface geometric model constructed by the isosurface, which results in a slower algorithm. This limits the application of the MC algorithm, so the academic community has proposed a lot of improved algorithms to solve this type problem. Montani et al. In [3] directly used the midpoint of the voxel edge instead of the intersection point of the isosurface and the cube voxel. This algorithm effectively reduced the calculation amount of the MC algorithm when calculating the vertices of the triangular patches. But the reconstruction results are inaccurate. The grid simplification algorithm is a representative improved algorithm to reduce the operation time [4]. But this algorithm improves the visual effect while improving. [5] is a commonly used algorithm that improves the traversal efficiency of voxels and effectively shortens the processing time of the MC algorithm. The Decimation algorithm reduced the number of triangles generated [6]. Xie $\mathrm{Z}$ et al. proposed an adaptive MC algorithm in [7], which greatly reduced the number of triangular patches, and the display effect was not much different from the standard algorithm. Liu et al. uses the tetrahedral partitioning method to reduce the number of triangular patches, which improves the speed of the rendering algorithm [8].

Aiming at the above problems, this paper proposes an improved MC algorithm. We first improve the representation of voxels. This reduces the number of voxels that the algorithm needs to traverse during the 3D reconstruction process and the 3D reconstruction time. Finally, the efficiency of the algorithm is improved. At the same time, based on the MC algorithm, smoothing and distortion coefficients are introduced. The smoothness and distortion of the 3D reconstruction model are controlled by setting the smoothing and distortion coefficients. Finally, before isosurface drawing, a mesh simplification algorithm based on quadratic measurement errors is introduced to reduce the output triangular mesh. The improved algorithm proposed in this paper responds faster to zoom, pan, and rotate operations in subsequent interactive applications. So it has better real-time interactivity.

The chapters of this article are arranged as follows. This article will first briefly introduce the basic principles of the MC algorithm in Section 2. In Section 3, an improved algorithm based on MC algorithm is introduced. The experimental comparison results of the traditional MC algorithm and the proposed algorithm are given in Section 4. We analyze the experimental results and give the advantages of the algorithm proposed in this paper. Finally, the full text is summarized in Section 5.

\section{MC Algorithm}

\subsection{Algorithm Overview}

MC algorithm is a kind of 3D reconstruction surface rendering algorithm. In 3D reconstruction of medical images, a CT image is a tomographic slice, and two adjacent CT images in the sequence are taken to form the upper and lower slices. The 2D CT tomographic image is described by 3D coordinates to obtain a 3D image of the human body. A 3D image or volume data can be described by a 3D array with corresponding values. Each of these elements becomes a volume element, referred to as a voxel. Each fault section takes four 
adjacent points, and a total of 8 points form a voxel. The basic idea of MC algorithm is to divide the 3D space into multiple voxels. Then iterate through each voxel to find a voxel that intersects the isosurface. Intersection points of voxels and isosurfaces are calculated by linear interpolation. Intersection points form triangle patches of different configurations within the voxels. The triangle patches are connected to complete the $3 \mathrm{D}$ reconstruction.

A voxel has 8 vertices, and each vertex has 2 states, in-plane and out-plane, so there are $2^{8}=256$ cases of the relationship between voxels and iso-surfaces. By enumerating these 256 cases, we can get a triangle patch configuration index table to record the information that the isosurface intersects with the sides of the voxel and the configuration of the triangle patch in the voxel. Utilizing the symmetry of the cube, the relationship between each vertex and surface on the surface and voxel is reversed at the same time, without affecting the configuration of the triangular patch in the voxel. In other words, the 256 cases are complementary to each other, so you only need to consider the cases where $0-4$ kinds of vertices are on the surface. In addition, according to the symmetry of the rotation in the three directions of the cube, the voxel is arbitrarily rotated by a multiple of 90 degrees in the three directions. The configuration of the triangular patch in the voxel is unchanged. Utilizing the characteristics of the above cube, 256 cases are finally reduced to 15 types of triangular patch configuration index table, as shown in Figure 1.
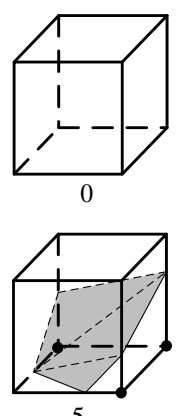

5

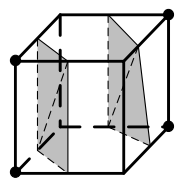

10
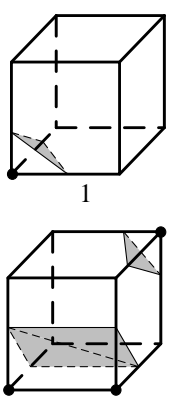

6

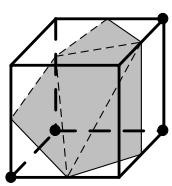

11
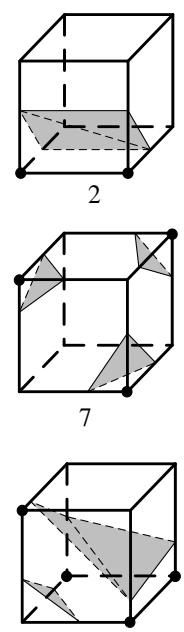

12
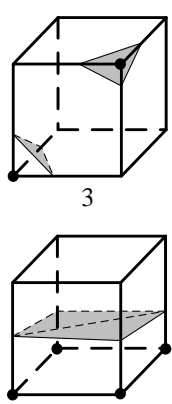

8

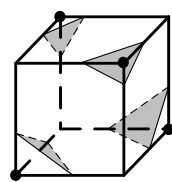

13
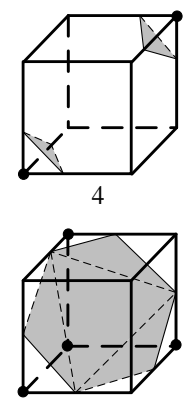

9

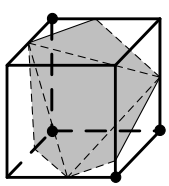

Fig. 1. Index table of 15 triangle patch configurations for voxels.

Each cube in Figure 1 represents a voxel. The numbers below the voxels represent the index values of the 15 triangle patch configurations. The shaded plane inside the cube represents the isosurface. The 15 cases in Figure $\mathbf{1}$ are arranged according to the number of voxel vertices in the isosurface from as few as possible. In each case, several triangle patches inside the voxel divide the voxel vertices into two categories, one inside the isosurface and the other outside the isosurface.

The key of MC algorithm is to find the isosurface in the original data. To find the isosurface with a threshold value of $c$, you must find the points where the gray value function 
value is $c$. These points are called equivalence points $(x, y, z)$. The isosurface can be expressed as a collection of isosurfaces, as shown in equation (1):

$$
\{(x, y, z) \mid F(x, y, z)=c\}
$$

where $c$ is the threshold of the isosurface, $c$ is a constant, $F$ is a gray value function, and the range of $F$ is $[0,255]$.

The equivalence points constitute several triangular patches. Then the triangular patches are fitted to the isosurface output. In order to display the isosurface image, the normal vector of each triangular patch forming the isosurface must be calculated. However, directly calculating the normal vector of each triangular patch is not only computationally intensive but also complicated. The MC algorithm uses gradient vectors instead of normal vectors. The gradient vector of each point on the isosurface represents the normal vector of the isosurface at that point. The normal vector $g(x, y, z)$ for a point $(x, y, z)$ in a 3D data field can be expressed as:

$$
g(x, y, z)=\nabla F(x, y, z)
$$

where $\nabla F(x, y, z)$ represents the gradient of the function $F$ at the point $(x, y, z)$.

The gradient of the vertices of the triangular patch is obtained by linear interpolation of the gradient of the voxels of the voxels. In actual calculation, the central difference method is used to calculate the gradient of each voxel vertex. For a certain voxel vertex $\left(x_{i}, y_{j}, z_{k}\right)$ in a 3D data field, its gradient $\nabla F\left(x_{i}, y_{j}, z_{k}\right)$ is calculated as:

$$
\nabla F\left(x_{i}, y_{j}, z_{k}\right)=\left(g_{x}, g_{y}, g_{z}\right)
$$

where $g_{x}, g_{y}, g_{z}$ represent the values of the normal vector in the $x, y$, and $z$ directions

$$
\begin{aligned}
& g_{x}=\frac{F\left(x_{i+1}, y_{j}, z_{k}\right)-F\left(x_{i-1}, y_{j}, z_{k}\right)}{2 \Delta x} \\
& g_{y}=\frac{F\left(x_{i}, y_{j+1}, z_{k}\right)-F\left(x_{i}, y_{j-1}, z_{k}\right)}{2 \Delta y} \\
& g_{z}=\frac{F\left(x_{i}, y_{j}, z_{k+1}\right)-F\left(x_{i}, y_{j}, z_{k-1}\right)}{2 \Delta z}
\end{aligned}
$$

where $\Delta x, \Delta y, \Delta z$ represent the side lengths of voxels in the $x, y$, and $z$ directions, $\left(x_{i+1}, y_{j}, z_{k}\right)$ and $\left(x_{i-1}, y_{j}, z_{k}\right)$ are voxel vertices adjacent to point $\left(x_{i}, y_{j}, z_{k}\right)$ in the $x$ direction, $\left(x_{i}, y_{j+1}, z_{k}\right)$ and $\left(x_{i}, y_{j-1}, z_{k}\right)$ are voxel vertices adjacent to point $\left(x_{i}, y_{j}, z_{k}\right)$ in the $y$ direction, $\left(x_{i}, y_{j}, z_{k+1}\right)$ and $\left(x_{i}, y_{j}, z_{k-1}\right)$ are voxel vertices adjacent to point $\left(x_{i}, y_{j}, z_{k}\right)$ in the $z$ direction.

\subsection{System Block Diagram of MC Algorithm}

The advantages of the MC algorithm are obvious, but it also has the following disadvantages. It can be known from the above analysis that in the MC algorithm, all voxels in the original 3D data need to be traversed. However, the number of voxels is often huge, which leads to a large amount of calculation and low efficiency of the MC algorithm. In addition, the 
calculation of the intersection between isosurface and voxel in MC algorithm uses a single interpolation method. 3D reconstruction cannot be performed according to user needs. The output of the MC algorithm is a triangular mesh. The data during 3D reconstruction is often very large. The number of $3 \mathrm{D}$ triangular meshes created in this way is huge, which greatly reduces the real-time interaction of the 3D reconstruction model in actual use.

Therefore, this paper proposes an improved CT image 3D reconstruction algorithm based on Marching Cubes algorithm. First, the CT image sequence is input as the original 3D data required for reconstruction. And select voxels to divide the original 3D data space. Then take out a certain voxel. The configuration of the triangular patch in the voxel is determined according to the triangular patch configuration index table. Calculate the coordinates of the equivalence points and the normal vector of the triangular patches. After traversing all the voxels in the 3D data space, all the obtained triangle patches are output to obtain isosurfaces. Finally, the triangle mesh of the isosurface is simplified to obtain the final 3D reconstruction model. This paper improves the traditional MC algorithm in three aspects: the voxel representation of the algorithm, the interpolation method and the simplification of the triangular mesh. The system block diagram of the improved algorithm is shown in Figure 2.

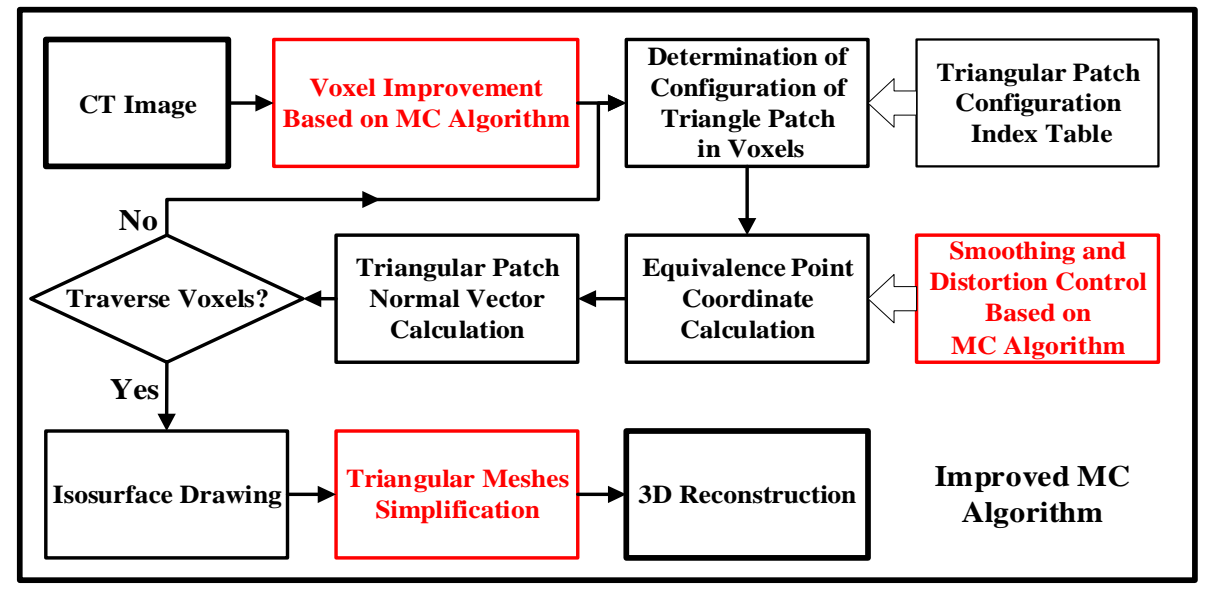

Fig. 2. Improved MC algorithm system block diagram.

In Figure 2, a CT image sequence is used as an input of a 3D reconstruction process. The 3D reconstruction model of the CT image is displayed as the final output result. Among them, the MC algorithm based voxel improvement algorithm will replace the original voxel selection process. The number of voxels to be traversed during the 3D reconstruction process is reduced to improve the operation efficiency of the 3D reconstruction algorithm. The smoothing and distortion coefficients are introduced in the calculation of the coordinates of the equivalent points by the MC algorithm to control the smoothness and distortion of the 3D reconstruction model. Finally, before the output of the 3D reconstruction model, a step of simplifying the triangular mesh is added to reduce the number of triangular meshes in the 3D reconstruction model. So that the 3D reconstruction model finally used in practical applications will have better real-time interaction. 


\section{Improvement of MC Algorithm}

\subsection{Improved Voxel Algorithm Based on MC Algorithm}

The two adjacent CT images in the CT sequence are taken out to form the upper and lower slices. Four points were taken from each of the upper and lower slices to form a voxel. A voxel is the basic unit of volume data. It can be regarded as a small cuboid with a certain size or abstracted as an abstract point in a space with certain attributes, as shown in Figure 3.

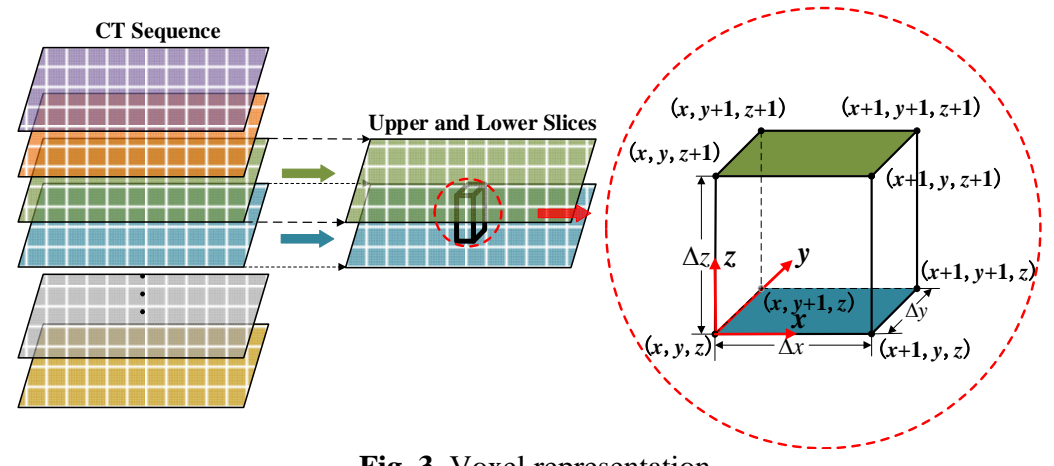

Fig. 3. Voxel representation.

In Figure 3, the 3D image or volume data can be represented by $\mathbf{I}(x, y, z, c)$, where $x, y$, and $z$ respectively represent coordinates in a $3 \mathrm{D}$ space, and $c$ represents a color component in a color image. When a certain dimension in a 3D image takes a fixed value, a 2D image can be obtained, which is commonly referred to as a tomographic image.

The traditional MC algorithm takes two adjacent images in the CT sequence to form the upper and lower slices. Each slice takes 4 adjacent points, and a total of 8 points form a voxel. Then the voxels need to be traversed, and the intersection coordinates need to be calculated by interpolation. Due to the huge number of voxels and the complicated calculation process of the interpolation method, the calculation amount of the MC algorithm is very large, and there will be a delay during 3D reconstruction. In order to improve the execution efficiency of the MC algorithm, this paper improves the voxel representation based on the MC algorithm. The improved voxel representation is shown in Figure 4.

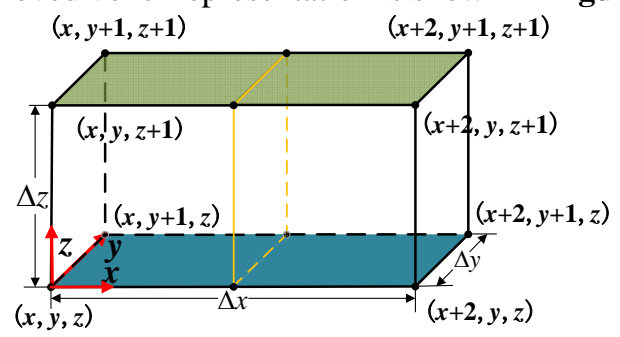

(a) Voxel representation with 2 pixels in the $\mathrm{x}$ direction

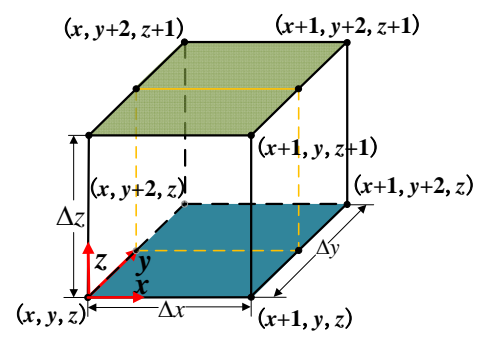

(b) Voxel representation with 2 pixels in the $y$ direction

Fig. 4. Improved voxel representation.

In the improved MC algorithm, the length of one side of the voxel is increased. As shown in Figure 4 (a) and Figure 4 (b), the side length of the voxel in the $x$ direction and the $y$ 
direction is improved to 2 pixels. The improved voxel volume is doubled to the original voxel volume. Therefore, the number of voxels to be traversed during the 3D reconstruction process is reduced to half, and the time required for reconstruction is greatly reduced. In terms of the influence of 3D reconstruction accuracy, the improved voxel representation can still control the error range within 2 pixels, which has little effect on the reconstruction accuracy. In order to further reduce the number of voxels that need to be traversed, this paper changes the voxel side length to 2 pixel lengths in the $x$ and $y$ directions at the same time, as shown in Figure 5. The improved voxel volume is four times the original. The number of voxels that need to be traversed is reduced to the original $1 / 4$, which further improves the operation efficiency of the proposed algorithm.

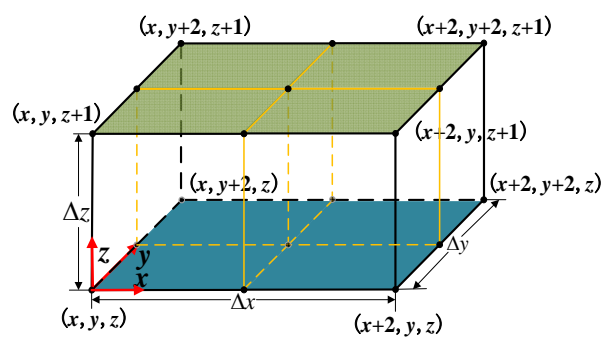

Fig. 5. Voxel representation with 2 pixels in the $x$ and $y$ directions.

After improving the representation of voxels, the original 3D data will be divided into individual voxels. Compare the relationship between the gray value of the voxel vertex and the isosurface threshold. Determine the state of each voxel vertex, in-plane or out-plane. After determining the state of the vertices, find the configuration corresponding to the voxel in the triangle patch configuration index table and record it. Next we need to calculate the coordinate values of the equivalence points. In calculating the coordinates of the equivalent points, in order to control the smoothness and distortion of the 3D reconstruction model, this paper introduces smoothness and distortion coefficients based on the MC algorithm.

\subsection{Smoothing and distortion control based on MC algorithm}

When calculating the coordinates of the iso-points, the MC algorithm first calculates the case where a voxel intersects the iso-surface, and obtains several triangle patches. Then iterate through all the voxels to get all the intersecting triangle patches. Finally, these triangular patches are fitted to the isosurface output. When computing the intersection of voxels and isosurfaces, the 3D spatial data of CT images are usually discrete. The data field along the voxel edge changes continuously and linearly. If the two vertices of an edge of a voxel are greater than or less than the value of the isosurface, there is only one intersection on the edge. If both vertices on an edge are larger or smaller than the value of the isosurface, the edge has no intersection with the isosurface. MC algorithm finds the intersection of isosurface and voxel by processing voxels in the data field one by one. Then we find the isosurfaces contained in these voxels.

The traditional MC algorithm uses linear interpolation to calculate the coordinates of the equivalent points. When using the linear interpolation method, it is necessary to assume that the function value of the discrete data field in 3D space changes linearly along the voxel 
boundary. Due to the existence of the above assumptions, the linear interpolation method makes the display effect of the 3D reconstruction model smoother.

Suppose the two endpoints $P_{i}=\left(x_{i}, y_{i}, z_{i}\right)^{\mathrm{T}}$ and $P_{j}=\left(x_{j}, y_{j}, z_{j}\right)^{\mathrm{T}}$ of the edge on which the equivalence points lie. $F$ represents the gray value function, then the gray values of endpoint $P_{i}$ and endpoint $P_{j}$ are $F_{i}=F\left(x_{i}, y_{i}, z_{i}\right)$ and $F_{j}=F\left(x_{j}, y_{j}, z_{j}\right)$. If the selected isosurface threshold is $c$, according to the basic principle of the MC algorithm, it can be known that the coordinates of any equivalence point $K=(x, y, z)^{\mathrm{T}}$ can be obtained by equation (5) through linear interpolation.

$$
\begin{aligned}
K & =\left(x_{i}, y_{i}, z_{i}\right)^{\mathrm{T}}+\frac{c-F_{i}}{F_{j}-F_{i}}\left(\left(x_{j}, y_{j}, z_{j}\right)^{\mathrm{T}}-\left(x_{i}, y_{i}, z_{i}\right)^{\mathrm{T}}\right) \\
& =\left(1-\frac{c-F_{i}}{F_{j}-F_{i}}\right)\left(x_{i}, y_{i}, z_{i}\right)^{\mathrm{T}}+\frac{c-F_{i}}{F_{j}-F_{i}}\left(x_{j}, y_{j}, z_{j}\right)^{\mathrm{T}}
\end{aligned}
$$

The median interpolation method is a simpler interpolation method. When calculating the coordinates of the equivalence points, it is only necessary to take the median of the coordinates corresponding to the edge where they are located. The median interpolation method can reduce distortion at the cost of smoothness. The median interpolation method is shown in equation (6):

$$
\begin{aligned}
K & =\left(x_{i}, y_{i}, z_{i}\right)^{T}+0.5\left(\left(x_{j}, y_{j}, z_{j}\right)^{T}-\left(x_{i}, y_{i}, z_{i}\right)^{T}\right) \\
& =0.5\left(x_{i}, y_{i}, z_{i}\right)^{T}+0.5\left(x_{j}, y_{j}, z_{j}\right)^{T}
\end{aligned}
$$

In order to control the smoothness and distortion of the 3D reconstruction model, parameter $\alpha$ is introduced in this paper to represent the smoothness and distortion coefficient. The formula for calculating the coordinates of the equivalence point by introducing parameter $\alpha$ is shown in equation (7).

$$
\begin{aligned}
K & =\left(x_{i}, y_{i}, z_{i}\right)^{\mathrm{T}}+\alpha\left(\frac{c-F_{i}}{F_{j}-F_{i}}-0.5\right)\left(\left(x_{j}, y_{j}, z_{j}\right)^{\mathrm{T}}-\left(x_{i}, y_{i}, z_{i}\right)^{\mathrm{T}}\right)+0.5\left(\left(x_{j}, y_{j}, z_{j}\right)^{\mathrm{T}}-\left(x_{i}, y_{i}, z_{i}\right)^{\mathrm{T}}\right) \\
& =\left(0.5-\alpha\left(\frac{c-F_{i}}{F_{j}-F_{i}}-0.5\right)\right)\left(x_{i}, y_{i}, z_{i}\right)^{\mathrm{T}}+\left(0.5+\alpha\left(\frac{c-F_{i}}{F_{j}-F_{i}}-0.5\right)\right)\left(x_{j}, y_{j}, z_{j}\right)^{\mathrm{T}}
\end{aligned}
$$

Control the smoothness and distortion of the 3D reconstruction model by changing $\alpha$ within the range of $[0,1]$. When $\alpha=0$, the interpolation method at this time becomes the median interpolation method. The 3D reconstruction model will have the lowest distortion. When $\alpha=1$, the interpolation method at this time becomes a linear interpolation method. The $3 \mathrm{D}$ reconstruction model will have the highest smoothness.

After the coordinates of the equivalent points are obtained, the triangle patches inside each voxel are determined according to the previously recorded triangle patch configuration. We get equivalent patches in the voxels. After outputting these isosurfaces together, the required isosurfaces are drawn.

The output of the MC algorithm is a triangular mesh. Generally speaking, these grids are huge and cannot meet the speed requirements of drawing and processing in interaction. In order to enhance the real-time interactivity of MC algorithm, this paper introduces a grid simplification algorithm based on quadratic measurement error to reduce the output triangular 
grid. The purpose of introducing measurement error is to quantify the difference between the input model and the output model. The simplification of the model is guided according to the measurement error, so that the simplification error is within the error range allowed by the user. The quadratic measurement error is recognized as a better measurement error. On the premise of maintaining the reliability of the original mesh, the number of triangular patches in the triangular mesh is reduced. This reduces the response time of the 3D reconstruction model in operations such as panning, zooming, and rotating.

Adding the simplified steps of the triangular mesh in the 3D reconstruction process can effectively reduce the number of meshes to be drawn without affecting the overall rendering effect, which is of great significance to both real-time rendering and real-time interaction.

\section{Experiment and Analysis}

\subsection{Efficiency Analysis of MC Algorithm Based on Improved Voxel Algorithm}

In order to illustrate the effectiveness of the above algorithm, this paper implements a 3D reconstruction algorithm and process of CT images based on the improved Marching Cubes algorithm. CT images are generally in DICOM format. In order to facilitate the reading of matlab, the DICOM format is converted to the .png format before 3D reconstruction. In order to facilitate comparison, two sets of abdominal enhanced CT images are selected as experimental data, and only three-dimensional reconstruction of liver tumors is performed. The pixel size of the two sets of CT images is $512 \times 512$. We tested our method on the competitive dataset of MICCAI LiTS Challenge[9]. The first set of reconstruction data consisted of 29 CT cut edges, and the second set consisted of 250 CT slices.

First, the voxel representations for 3D reconstruction were selected, and 3 voxel representations were used to perform 3D reconstruction of the liver tumor surface. The first voxel representation is the voxel representation of the traditional MC algorithm, as shown in Figure 1. The second voxel is represented as an improved voxel representation, as shown in Figure 5. The third voxel is represented as an improved voxel representation, and the voxel sides in the $\mathrm{x}$ and $\mathrm{y}$ directions are both 3 pixels.

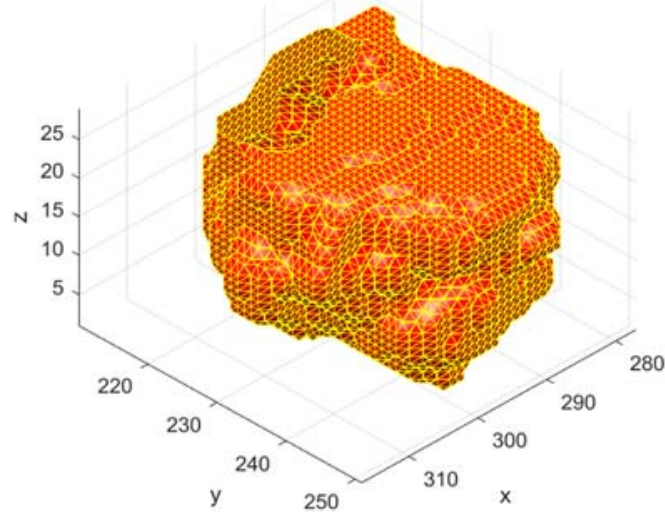

(a) Reconstruction of MC algorithm

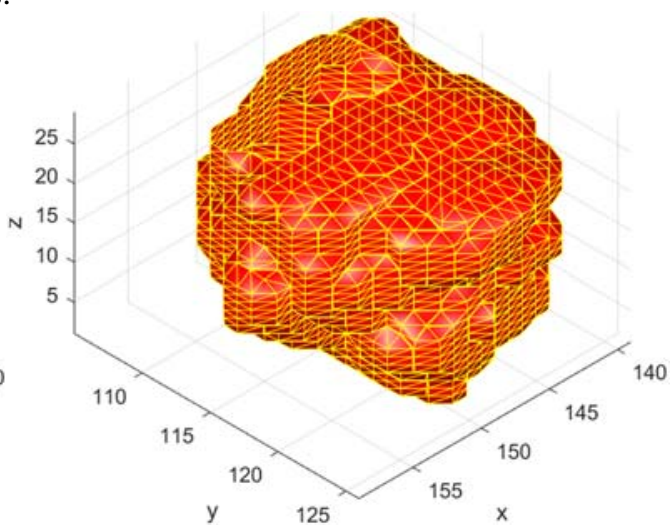

(b) Reconstruction with voxel sides of 2 pixels

Fig. 6. Reconstruction results of the first set of data. 


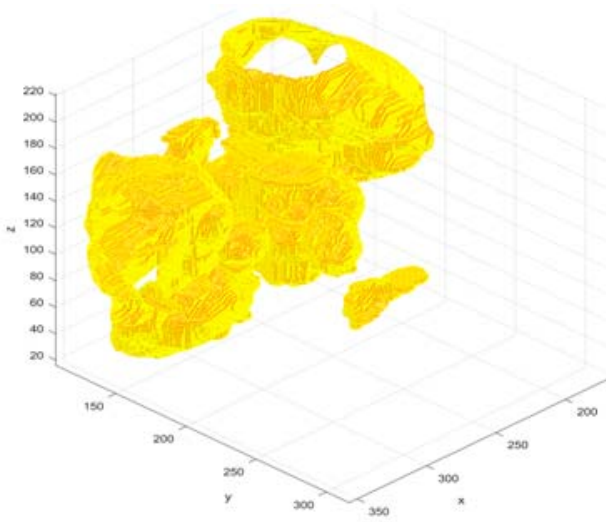

(a) Reconstruction of MC algorithm

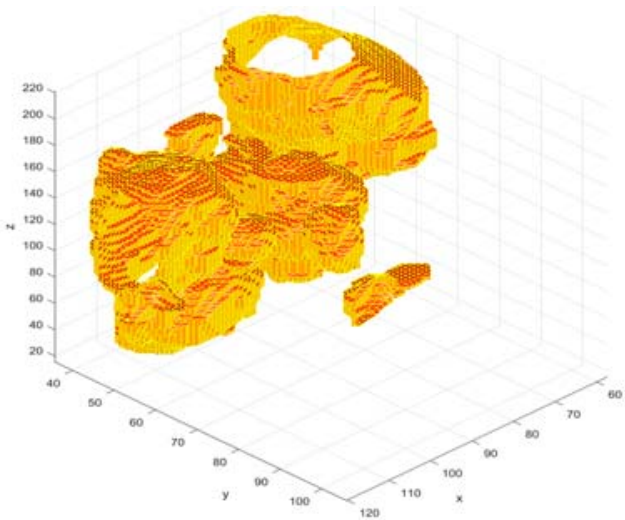

(b) Reconstruction with voxel sides of 3 pixels

Fig. 7. Reconstruction results of the second set of data.

Figure 6 shows the results of processing the first set of reconstruction data, which can clearly reconstruct the surface of a single liver tumor. The reconstructed tumor surface consists of several triangular patches. In order to facilitate observation, the surface of the triangular patch is shown in red in this figure. The borders of the triangles are drawn in yellow. As can be seen from the figure, compared with the traditional MC algorithm, the proposed algorithm has a larger area and a smaller number of triangles.

In Figure 7, a three-dimensional reconstruction is performed using the second set of reconstruction data. The entire 3D reconstruction model is yellow. The second set of data has a larger amount of data than the first set of data, making the displayed triangular patches denser. The red surface area is compressed when displayed, and the yellow border is more prominent. From the overall effect of reconstruction, the MC algorithm based on voxel changes has little effect on the accuracy of 3D reconstruction.

Table 1. Efficiency comparison of reconstruction algorithms

\begin{tabular}{|c|c|c|c|c|}
\hline \multirow[b]{2}{*}{ Voxel representation } & \multicolumn{2}{|c|}{ The first set of data } & \multicolumn{2}{|c|}{ The second set of data } \\
\hline & $\begin{array}{l}\text { Reconstruction } \\
\text { time/s }\end{array}$ & $\begin{array}{l}\text { Improved } \\
\text { efficiency }\end{array}$ & $\begin{array}{l}\text { Reconstruction } \\
\text { time/s }\end{array}$ & $\begin{array}{l}\text { Improved } \\
\text { efficiency }\end{array}$ \\
\hline $\begin{array}{l}\text { Voxel representation of } \\
\text { MC algorithm }\end{array}$ & 0.809010 & & 27.291630 & \\
\hline $\begin{array}{l}\text { Voxel representation with } 2 \text { pixels } \\
\text { in the } \mathrm{x} \text { and } \mathrm{y} \text { directions }\end{array}$ & 0.314780 & $61.0907 \%$ & 1.763029 & $93.5400 \%$ \\
\hline $\begin{array}{l}\text { Voxel representation with } 3 \text { pixels } \\
\text { in the } \mathrm{x} \text { and } \mathrm{y} \text { directions }\end{array}$ & 0.169684 & $79.0257 \%$ & 0.867009 & $96.8232 \%$ \\
\hline
\end{tabular}

By comparing the reconstruction time, it can be seen from Table 1 that no matter for the reconstruction of the first or second set of data, the operation efficiency of the MC algorithm based on voxel changes is much greater than the traditional MC algorithm. This is because when the side length of the voxel becomes longer, the number of voxels traversed during the $3 \mathrm{D}$ reconstruction process will be reduced. Therefore, the time of 3D reconstruction is greatly reduced, and the operation efficiency of the 3D reconstruction algorithm is greatly improved. 
When the amount of data that needs to be reconstructed is larger, the difference in visual perception between the results of the proposed algorithm and the traditional MC algorithm is smaller, and the efficiency of the algorithm is significantly improved.

\subsection{Effect Analysis of MC Algorithm with Smoothing and Distortion Coefficients}

Three-dimensional reconstruction is performed on the same set of data under different smoothing and distortion coefficients. The reconstruction effect is compared. As shown in Figure 8, the smoothness and distortion can be controlled by introducing smoothness and distortion coefficients.

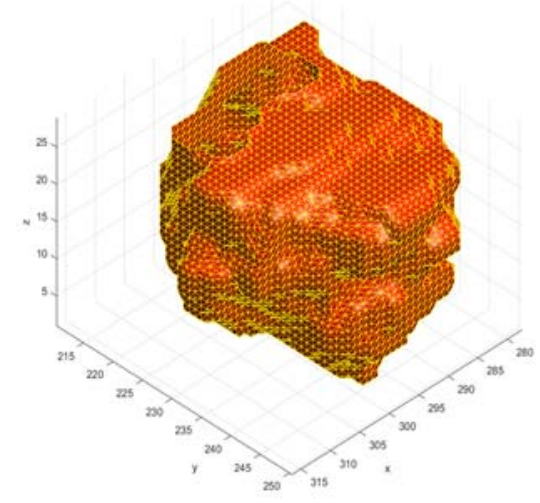

(a) smoothing distortion coefficient $=0$

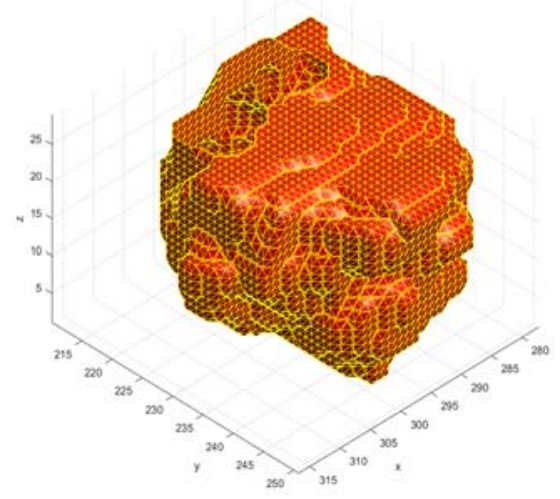

(c) smoothing distortion coefficient $=0.75$

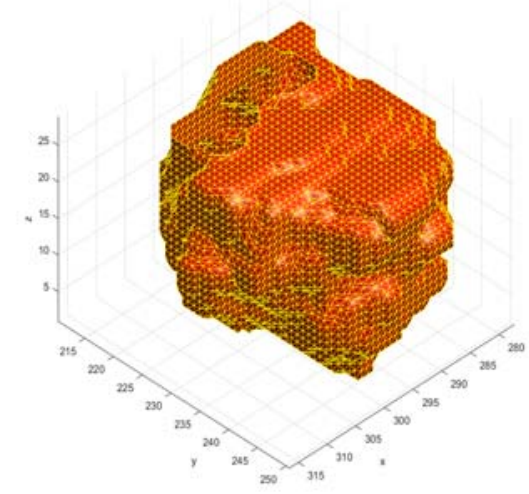

(b) smoothing distortion coefficient $=0.25$

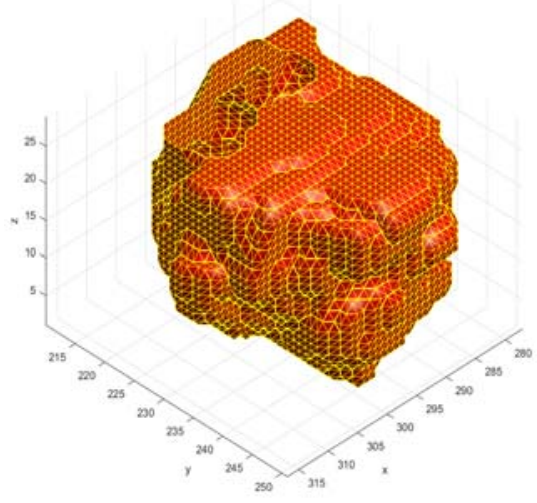

(d) smoothing distortion coefficient $=1$

Fig. 8. Reconstruction results with different smoothing and distortion coefficients.

In Figure 8(a), under the condition that the smoothing distortion coefficient is equal to 0, the interpolation method at this time becomes the median interpolation method. It can be seen from the figure that the triangles that make up the isosurface are of uniform size, consistent shape, and neat orientation. The 3D reconstruction model is more rigid in visual effects and the smoothness is reduced. In Figure 8(b) and Figure 8(c), the smoothing and distortion coefficients are set to 0.25 and 0.75 . In Figure 8(d), under the condition that the smooth distortion coefficient is equal to 1 , the interpolation method at this time becomes a linear 
interpolation method. It can be seen from the figure that whether on a flat surface or an uneven surface, the triangular patches that make up the isosurface will change in size, shape and direction according to the gradient of the gray value. The smoothness of the 3D reconstruction model is improved, but this is at the cost of the distortion of the 3D reconstruction model.

\subsection{Effect Analysis of MC Algorithm with Smoothing and Distortion Coefficients}

The experiment uses the first set of reconstruction data for 3D reconstruction. Before the isosurface is drawn, a simplified step of adding a triangle mesh is added. This paper chooses the rotation operation to test the real-time interactivity of the 3D reconstruction model. The 3D reconstruction model is continuously rotated, each rotation angle is $20^{\circ}$, the azimuth angle is changed from $0^{\circ}$ to $360^{\circ}$, and a circle around the $\mathrm{z}$ axis. The experimental results are shown in Table 2.

Table 2. Comparison of rotation time

\begin{tabular}{ccccccc}
\hline & $\begin{array}{c}\text { First } \\
\text { time/s }\end{array}$ & $\begin{array}{c}\text { Second } \\
\text { time/s }\end{array}$ & $\begin{array}{c}\text { Third } \\
\text { time/s }\end{array}$ & $\begin{array}{c}\text { Fourth } \\
\text { time/s }\end{array}$ & $\begin{array}{c}\text { Fifth } \\
\text { time/s }\end{array}$ & $\begin{array}{c}\text { Average } \\
\text { time/s }\end{array}$ \\
\hline Not simplified & 4.000108 & 3.876800 & 3.749688 & 4.242814 & 3.701339 & 3.91415 \\
Simplification rate of \\
$\begin{array}{c}50 \% \\
\text { Simplification rate of } \\
90 \%\end{array}$
\end{tabular}

In order to make the experimental results more accurate, this article has measured the operating time several times. The average value is calculated for the analysis of the simplified effect of the triangular mesh. In this paper, the continuous rotation time is recorded in three cases: unsimplified, $50 \%$ simplified and $90 \%$ simplified. As can be seen from Table 2, the higher the simplification rate of the triangular mesh, the shorter the time taken for the continuous rotation operation. When the simplification rate is $50 \%$, the average time of continuous rotation operation is reduced by $11.86 \%$ compared with the case without simplification. When the simplification rate is $90 \%$, the average time of continuous rotation operation is even reduced by $25.63 \%$. It can be verified that the triangular mesh simplification can well enhance the real-time interactivity of the 3D reconstruction model.

\section{Conclusion}

Aiming at the problems of low efficiency of 3D reconstruction algorithm of CT image and insufficient practicality of 3D reconstruction model, this paper proposes an improved algorithm based on MC. First, by improving the representation of voxels, the volume of voxels can be increased. This reduces the number of voxels that need to be traversed during the 3D reconstruction process, and further improves the operation efficiency of the 3D reconstruction algorithm. At the same time, smoothing and distortion coefficients are introduced in the process of calculating the equivalent points to control the smoothness and distortion of the 3D reconstruction model. Before drawing the isosurface, the triangulated mesh based on the quadratic measurement error is used to reduce the output triangular mesh. The real-time 
interaction of the 3D reconstructed model is enhanced, so that the model has a faster response speed in the process of translation, enlargement and rotation.

Acknowledgments. This paper is supported by national nature scinece foundation of China (61971162, 41861134010).

\section{References}

[1] Lorensen W E, Cline H E. Marching cubes: a high resolution 3D surface construction algorithm[M]// Seminal graphics. ACM, 1998.

[2] Newman T S, Yi H. A survey of the marching cubes algorithm[J]. Computers \& Graphics, 2006, 30(5):854-879.

[3] Montani C, Scateni R, Scopigno R . Discretized Marching Cubes[C]// Visualization, 1994. Visualization '94, Proceedings. IEEE Conference on. IEEE, 1994.

[4] Wang Z L , Teo J C M , Chui C K , et al. Computational biomechanical modelling of the lumbar spine using marching-cubes surface smoothened finite element voxel meshing[J]. Comput Methods Programs Biomed, 2005, 80(1):25-35.

[5] Zhang Y , Qian J . Dual Contouring for domains with topology ambiguity[J]. Computer Methods in Applied Mechanics and Engineering, 2012, 217-220(none):34-45.

[6] Li W , Chen Y , Wang Z , et al. An improved decimation of triangle meshes based on curvature.[C]// International Conference on Rough Sets \& Knowledge Technology. Springer, Cham, 2014.

[7] Xie Z , Zhao Y , Fan X , et al. The research of extending marching cubes with adaptive methods based on volume warping[J]. Zhongshan Daxue Xuebao/Acta Scientiarum Natralium Universitatis Sunyatseni, 2012, 51(5):67-72.

[8] Liu Y , Xing H L . A boundary focused quadrilateral mesh generation algorithm for multi-material structures[J]. Journal of Computational Physics, 2013, 232(1):516-528.

[9] https://competitions.codalab.org/competitions/17094. 